\title{
Babinet Principle Applied to the Design of Metasurfaces and Metamaterials
}

\author{
F. Falcone,${ }^{1, *}$ T. Lopetegi, ${ }^{1}$ M. A. G. Laso, ${ }^{1}$ J. D. Baena, ${ }^{2}$ J. Bonache,${ }^{3}$ M. Beruete,${ }^{1}$ \\ R. Marqués, ${ }^{2}$ F. Martín, ${ }^{3}$ and M. Sorolla ${ }^{1}$ \\ ${ }^{1}$ Departamento de Ingeniería Eléctrica y Electrónica, Universidad Pública de Navarra, 31006-Pamplona, Spain \\ ${ }^{2}$ Departamento de Electrónica y Electromagnetismo, Universidad de Sevilla, 41012-Sevilla, Spain \\ ${ }^{3}$ Departament d'Enginyeria Electrónica, Universitat Autónoma de Barcelona, 08193-Barcelona, Spain
}

(Received 16 February 2004; published 1 November 2004)

\begin{abstract}
The electromagnetic theory of diffraction and the Babinet principle are applied to the design of artificial metasurfaces and metamaterials. A new particle, the complementary split rings resonator, is proposed for the design of metasurfaces with high frequency selectivity and planar metamaterials with a negative dielectric permittivity. Applications in the fields of frequency selective surfaces and polarizers, as well as in microwave antennas and filter design, can be envisaged. The tunability of all these devices by an applied dc voltage is also achievable if these particles are etched on the appropriate substrate.
\end{abstract}

DOI: 10.1103/PhysRevLett.93.197401

PACS numbers: 78.20.Ci, 41.20.Jb, 42.25.Fx, 84.40.-x

Artificial metamaterials and metasurfaces with special electromagnetic properties have been a subject of growing interest in recent years [1,2]. Most proposed metamaterials make use of split ring resonators (SRRs) [3], or similar geometries, to achieve a negative effective permeability in a certain frequency range. The negative permittivity has been commonly obtained from an array of metallic wires or plates $[2,4]$. No particles acting as point electric dipoles with negative polarizability have been proposed to the date. In addition to these bulk metamaterial designs, one- and two-dimensional planar microwave circuits which show a left-handed behavior have been recently proposed [5-7], some of them making use of the SRR concept [7]. More recently, the application of these concepts to the design of artificial surfaces with special electromagnetic properties has been considered [8].

In this Letter we present a new approach for the design of planar metamaterials and metasurfaces, which is based on the Babinet principle. The key element of this new approach is the complementary split ring resonator (CSRR), the complementary screen of the SRR (see Fig. 1). As a first step in our analysis the behavior of a perfectly conducting and infinitely thin SRR in an external electromagnetic field $\mathbf{E}^{0}, \mathbf{B}^{0}$ [see Fig. 2(a)] is considered. The scattered field $\mathbf{E}^{\prime}, \mathbf{B}^{\prime}$ is approximately given by the field produced by a resonant magnetic dipole [3]

$$
\mathbf{m}=\alpha_{0}\left(\omega_{0}^{2} / \omega^{2}-1\right)^{-1} \mathbf{B}^{0} \cdot \hat{\mathbf{z}} \hat{\mathbf{z}},
$$

where $\omega_{0}$ is the frequency of resonance of the SRR and $\alpha_{0}$ is a geometrical factor. This approximation neglects higher order multipolar fields [2,3]. It also neglects cross-polarization effects $[9,10]$ (these effects are considered later in this Letter). Let us now consider the behavior of the CSRR when it is illuminated from $z<0$ by an external electromagnetic field $\mathbf{E}_{c}^{0}, \mathbf{B}_{c}^{0}$ [see Fig. 2(b)].
According to the electromagnetic theory of diffraction [11], the field in the shadowed region $(z>0)$ is the field scattered by the CSRR, $\mathbf{E}_{c}^{\prime}, \mathbf{B}_{c}^{\prime}$. For $z<0$, the total field is given by [11]

$$
\mathbf{E}_{c}=\mathbf{E}_{c}^{0}+\mathbf{E}_{c}^{0, r}+\mathbf{E}_{c}^{\prime} ; \quad \mathbf{B}_{c}=\mathbf{B}_{c}^{0}+\mathbf{B}_{c}^{0, r}+\mathbf{B}_{c}^{\prime},
$$

where $\mathbf{E}_{c}^{0, r}, \mathbf{B}_{c}^{0, r}$ is the field that would be reflected by the metallic screen without the CSRRs etched on it. The scattered fields, $\mathbf{E}_{c}^{\prime}, \mathbf{B}_{c}^{\prime}$ and $\mathbf{E}^{\prime}, \mathbf{B}^{\prime}$, must fulfill some symmetries that arise from the fact that they are produced by currents which are confined in the $z=0$ plane: the components $B_{z}^{\prime}, E_{x}^{\prime}$, and $E_{y}^{\prime}$ must be even functions of $z$, while $E_{z}^{\prime}, B_{x}^{\prime}$, and $B_{y}^{\prime}$ must be odd functions of the same variable [11].

According to the Babinet principle, if a screen with apertures (the CSRR) is illuminated from $z<0$ by an incident field $\mathbf{E}_{c}^{0}, \mathbf{B}_{c}^{0}$ and its complementary screen (the SRR) is illuminated by some complementary incident fields $\mathbf{E}^{0}=c \mathbf{B}_{c}^{0}, \mathbf{B}^{0}=-(1 / c) \mathbf{E}_{c}^{0}$, then at the shadowed side $(z>0)$ the total fields must satisfy [11]

$$
\mathbf{E}_{c}-c \mathbf{B}=\mathbf{E}_{c}^{0} ; \quad c \mathbf{B}_{c}+\mathbf{E}=\mathbf{B}_{c}^{0} .
$$

Assuming that the SRR scattered field is described by (1),
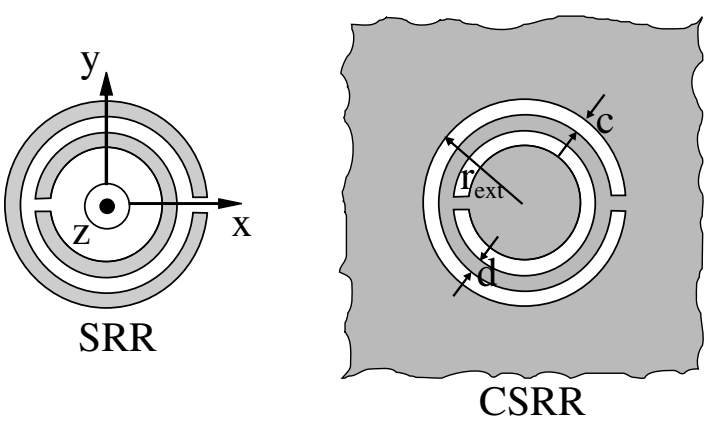

FIG. 1. Geometries of the SRR and the CSRR. 


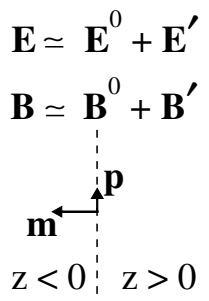

(a)

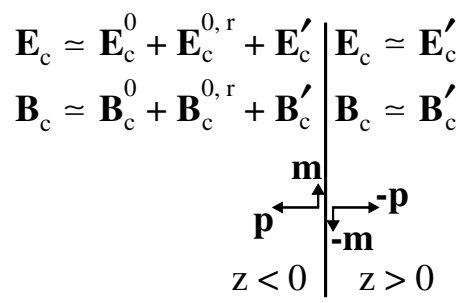

(b)
FIG. 2. Illustration of the behavior of a SRR (a) and a CSRR (b) when they are illuminated by an external field coming from $z<0$. Big arrows account for the main excitation mechanism [3] given by (1) and (5). Small arrows stands for the cross-polarization effect $[9,10]$.

it can be easily verified that in order to satisfy (3), the fields scattered by the CSRR at $z>0, \mathbf{E}_{c}^{\prime}, \mathbf{B}_{c}^{\prime}$ should be those produced by an electric dipole $\mathbf{p}=(1 / c) \mathbf{m}$, or

$$
\mathbf{p}=-\frac{1}{c^{2}} \alpha_{0}\left(\omega_{0}^{2} / \omega^{2}-1\right)^{-1} \mathbf{E}_{c}^{0} \cdot \hat{\mathbf{z}} \hat{\mathbf{z}}
$$

In the nonshadowed region $(z<0)$ the sign of this dipole must change, in order to produce the aforementioned symmetry properties of the scattered fields. Thus, for $z<0$ we finally obtain

$$
\mathbf{p}=\alpha_{0, c}\left(\omega_{0}^{2} / \omega^{2}-1\right)^{-1} \mathbf{E}_{c}^{0} \cdot \hat{\mathbf{z}} \hat{\mathbf{z}} ; \quad \alpha_{0, c}=\left(1 / c^{2}\right) \alpha_{0},
$$

where $c$ is the velocity of light in vacuum. For lossy and/ or thick screens, as well as in conventional circuit boards, the previous equations should be considered only as an approximation. Since $\mathbf{E}_{c}^{0}+\mathbf{E}_{c}^{0, r}=2 \mathbf{E}_{c}^{0} \cdot \hat{\mathbf{z}} \hat{\mathbf{z}}$ at $z=0^{-}$, it may be convenient, for some applications, to substitute (5) by

$$
\mathbf{p}=\beta_{0}\left(\omega_{0}^{2} / \omega^{2}-1\right)^{-1} \mathbf{E}_{\mathrm{ext}},
$$

where $\beta_{0}=\alpha_{0, c} / 2$ and $\mathbf{E}_{\text {ext }}=\mathbf{E}_{c}^{0}+\mathbf{E}_{c}^{0, r}$ is the total external field produced by the sources and the metallic screen without the CSRR.

The aforementioned results can be directly applied to the design of artificial metasurfaces. In fact, the results for a single CSRR can be extended to a system of many CSRRs with a density of $N$ CSRRs per square meter. Since the CSRRs are electrically small [3], the distance between them can be made much smaller than the incident radiation wavelength. Thus we are in the long wavelength limit, and the considered metallic surface can be seen - from the source side - as an electric dipolar sheet of magnitude $\mathbf{P}_{s}=\frac{1}{2} N \mathbf{p}$ on top of a flat metallic screen. From the opposite side, a dipolar sheet of the same intensity but of opposite sign is seen [see Fig. 2(b)]. Let us consider the incidence of a plane wave on a CSRR metasurface. Assuming that the angle of incidence and the polarization of this wave allows for the CSRRs exci- tation, the induced dipolar sheet also produces an electromagnetic wave which interferes with the plane wave reflected at the metallic screen. The effect of this interference can be dramatic near the resonance $\omega_{0}$. In particular, the CSRRs screen could be potentially tailored in order to destroy the reflected wave. At this frequency all the electromagnetic power will be transmitted through the screen. This behavior is just the dual of that expected for a surface of $N$ SRRs per square meter, illuminated by the complementary wave. In this case, a magnetic dipolar sheet is produced which, eventually, cancels the transmitted wave, thus reflecting all the incident power.

Up to now, the cross-polarization effects in the SRR $[9,10]$ have been neglected. In the frame of such an approximation, the aforementioned effects in a multiple CSRR (SRR) screen would appear only if there is a normal component of the electric (magnetic) incident field, because only in this case the CSRRs (SRRs) are excited. This fact would prevent the excitation of a multiple SRR (CSRR) metasurface by a normally incident plane wave, thus limiting its usefulness as frequency selective surfaces. However, when cross-polarization effects are considered [9,10], it becomes apparent that a similar excitation can be observed for incident fields with a nonvanishing component of the electric field along the $y$ axis of the SRRs (see Fig. 1). From duality, a similar behavior is expected for CSRRs illuminated by an external magnetic field polarized along the same axis. Electromagnetic simulations (using the CST Microwave Studio electromagnetic solver) have confirmed this hypothesis. An experimental setup has been also designed and built up to prove such effects. The experimental device consists of a pair of CSRRs and SRRs metasurfaces which were illuminated from one side by a normally incident plane wave. The transmitted field was measured at the opposite side (two horn antennas were used for these purposes). Both metasurfaces were etched on a commercial low loss microwave substrate (Arlon 250 $L X-0193-43-11$, whose parameters are shown in the caption of Fig. 3). The measured transmission coefficients for the appropriate polarization of the incident wave - magnetic (electric) field along the $y$ axis for the CSRR (SRR) metasurface - are shown in Fig. 3. A sharp transmission peak can be observed for the CSRR metasurface at the resonance, whereas a sharp null can be also observed in the SRR metasurface at a similar frequency. These results confirm the proposed theory (the frequency of resonance of the SRRs predicted by the model reported in [12] is $\omega_{0} \simeq 2 \pi 4.41 \times 10^{9} \mathrm{~s}^{-1}$ ). The shift between the frequency of resonance of the SRR and the CSRR metasurfaces can be mainly attributed to the effect of the dielectric substrate, which affects in a different way the frequency of resonance of the SRRs and the CSRRs. The limited height of the transmission peak for the CSRR metasurface can be attributed to the presence of the 


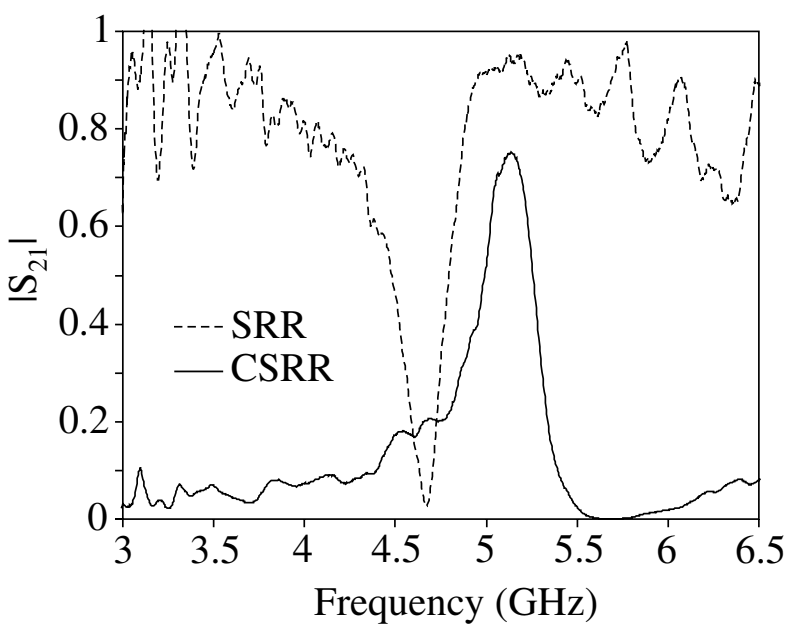

FIG. 3. Measured transmission coefficient through a CSRR (solid line) and a SRR (dashed line) frequency selective surface. The CSRRs or SRRs are etched on a commercial low loss microwave board with permittivity $\epsilon=2.43 \epsilon_{0}$, thickness $h=$ $0.49 \mathrm{~mm}$, and metallizations of copper with a thickness $t=$ $35 \mu \mathrm{m}$. The CSRR and SRR parameters are (see Fig. 1) $r_{\mathrm{ext}}=$ $3.5 \mathrm{~mm}, c=0.4 \mathrm{~mm}$, and $d=0.4 \mathrm{~mm}$. The CSRRs and SRRs are arranged in a square lattice with periodicity $a=8 \mathrm{~mm}$. The characteristics of the incident waves are described in the text.

substrate, the finite size of the metasurface, and Ohmic losses (simulations in the absence of the substrate and neglecting losses show a $100 \%$ of transmission). As expected, no effect was observed for the orthogonal polarization, thus showing that a multiple CSRR or SRR metasurface also acts as a polarizer. Subsequent simulations of transmission through thick (up to $5 \mathrm{~mm}$ ) perfectly conducting metallic screens have shown that this thickness significantly affects the location of the transmission maxima but does not destroy the reported qualitative effect. Similar simulations show that the location of the maxima is not significantly affected by the angle of incidence, nor by the CSRR periodicity. This independence of the maxima location with the angle of incidence and CSRR periodicity is in complete agreement with the proposed theory: the resonance is mainly a property of the individual CSRRs, not a characteristic of the array.

Another application of the reported concepts is in the design of planar metamaterials. Most of present high frequency passive circuits are designed in planar technology, so that the electromagnetic field is confined between a metallic ground plane and some metallic strips or patches, with a dielectric substrate filling the interspacing. Let us suppose that some CSRRs are etched on the ground plane at regions where the electromagnetic field is significant. The effect of these CSRRs is to produce an additional polarization in this region. According to (6), this polarization is simply proportional to the external field, and highly negative (that is, antiparallel to this field) around and above the frequency of resonance.
Thus, the effect of the CSRRs is to change the effective permittivity of the substrate from positive to negative values in some frequency region near the frequency of resonance of the CSRRs. Therefore, a substrate region of effective negative permittivity can be created by this procedure.

In order to show this effect by an experiment, the planar structure schematized in Fig. 4 has been designed and manufactured. This is a modification of the wellknown microstrip transmission line, incorporating some CSRRs etched on the ground plane, underneath the upper strip. Capacitive gaps can be also present on the strip. According to the previous theory, a negative effective dielectric permittivity will appear in the substrate region of interest, near the CSRRs resonance. Thus, microwave propagation will be inhibited in this frequency range. In order to switch this stop band behavior to a bandpass one (i.e., microwave propagation only at those frequencies where $\epsilon_{\text {eff }}<0$ ), we can resort to a strategy well known by electrical engineers: by periodically loading the microstrip line with capacitors, the inductive series impedance of the strip line is changed to a capacitive one, thus allowing propagation on negative $\epsilon$ substrates. This is just the expected effect of the capacitive gaps etched on the microstrip. It is also worth noting that the resulting transmission line has a backward-wave behavior and can be considered as one dimensional analogous of a left-handed medium [13].

The practical device was manufactured on a commercial low loss microwave dielectric board (Rogers RO-3010), and the device parameters are shown in the caption of Fig. 5. The strip line was chosen in order to obtain the usual characteristic impedance of $50 \Omega$ for the host line. Finally, the capacitive gaps - when present have been designed in order to introduce a per unit length series capacitance which dominates, in the frequency range of interest, over the line inductance. Figure 5 shows the simulated and measured transmission coefficient for the four-stage microstrip transmission line shown in Fig. 4, with and without capacitive gaps etched on the metallic strip. A good agreement can be found between the experiment and the simulations, and discrepancies can be attributed to Ohmic losses in the CSRRs (losses were neglected in the simulations). A passband appears for the

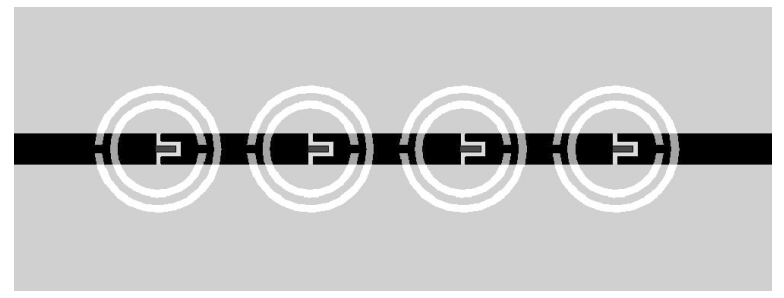

FIG. 4. A microstrip line (in black) over a ground plane (in gray) with some CSRRs etched on it. Capacitive gaps have been etched on the strip in order to obtain a left-handed passband. 


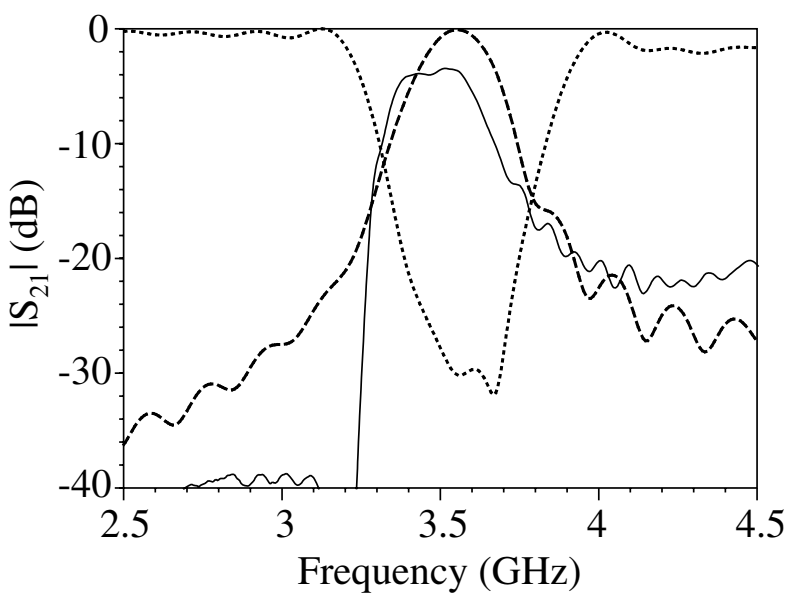

FIG. 5. Simulated results (using CST Microwave Studio) for the transmission coefficient in the structure of Fig. 4 with (dashed line) and without (dotted line) capacitive gaps. The experimental results for the transmission coefficient with capacitive gaps are also shown (solid line). Substrate parameters are $\epsilon=10.2 \epsilon_{0}, h=1.27 \mathrm{~mm}$, with a copper layer of $35 \mu \mathrm{m}$ thick. The CSRR parameters are $r_{\text {ext }}=2.5 \mathrm{~mm}$ and $c=d=$ $3 \mathrm{~mm}$. The strip width is $w=1.2 \mathrm{~mm}$.

line with CSRRs and capacitive gaps at the same frequencies where a stop band can be observed for the line without the gaps. We interpret these results as a consequence of the aforementioned effective negative dielectric permittivity of the substrate in such frequency range. This interpretation is confirmed by the dispersion relation of the line periodically loaded with CSRRs and capacitive gaps, which has been computed from the transmission coefficient of a single unit cell. The results of these simulations (not explicitly shown due to the limited space of this Letter) evidence a backward-wave behavior, in agreement with the proposed theory. In contrast to SRRbased structures, the CSRR structures have a connected metallic ground plane which extend over all the structure. This last characteristic makes easy the tuning of the structure if dielectric substrates with a significant dependence of its permittivity on an external electrostatic field [14] are used. For instance, in the modified transmission line shown in Fig. 4, it is enough to apply an external dc voltage between the strip line and the ground to obtain this tuning. Simulations have shown a high sensitivity of the location of the transmission peak of Fig. 5 to variations of the substrate permittivity.

In summary, the Babinet principle has been applied to the design of artificial surfaces and materials with extraordinary electromagnetic properties. A new particle, the CSRR, which acts as an electric point dipole with negative polarizability, has been proposed following this approach. This concept goes beyond the usual approach for making negative- $\epsilon$ metamaterials, based on the use of metallic wires or plates. It has been shown that by etching CSRRs in a common metallic plate, artificial metasurfaces with special electromagnetic transmission properties can be designed. Artificial planar metamaterials with negative $\epsilon$ can be also designed by a similar procedure. By properly combining these designs with other elements providing an effective negative magnetic permeability, a left-handed behavior is achieved. Practical application of these concepts in the design of frequency selective surfaces and polarizers, as well as antennas, filters, and other microwave devices, is envisaged. These applications will take advantage of the small electrical size of the CSRRs (and SRRs) at resonance, thus resulting in a significant miniaturization.

This work has been supported by the Spanish Ministry of Science and Technology and FEDER funds by Project Contracts No. TEC2004-04249-C02-01, No. TEC200404249-C02-02, and No. TIC2002-04528-C02-01.

*Electronic address: falcone_fj@tsm.es

[1] L. Martin-Moreno, F. J. Garcia-Vidal, H. J. Lezec, K. M. Pellerin, T. Thio, J. B. Pendry, and T.W. Ebbesen, Phys. Rev. Lett. 86, 1114 (2001).

[2] D. R. Smith, W. J. Padilla, D. C. Vier, S. C. Nemat- Nasser, and S. Schultz, Phys. Rev. Lett. 84, 4184 (2000).

[3] J. B. Pendry, A. J. Holden, D. J. Robbins, and W. J. Stewart, IEEE Trans. Microwave Theory Tech. 47, 2075 (1999).

[4] R. Marqués, J. Martel, F. Mesa, and F. Medina, Microwave Opt. Tech. Lett. 35, 405 (2002).

[5] G.V. Eleftheriades, A. K. Iyer, and P.C. Kremer, IEEE Trans. Microwave Theory Tech. 50, 2702 (2002).

[6] A. Grbic and G. V. Eleftheriades, J. Appl. Phys. 92, 5930 (2002).

[7] F. Martín, J. Bonache, F. Falcone, M. Sorolla, and R. Marqués, Appl. Phys. Lett. 83, 4652 (2003).

[8] T. J. Yen, W. J. Padilla, N. Fang, D. C. Vier, D. R. Smith, J. B. Pendry, D. N. Basov, and X. Zhang, Science 303, 1494 (2004).

[9] R. Marqués, F. Medina, and R. Rafii-El-Idrissi, Phys. Rev. B 65, 144440 (2002).

[10] Ph. Gay-Balmaz, and O. J. F. Martin, J. Appl. Phys. 92, 2929 (2002).

[11] J. D. Jackson, Classical Electrodynamics (Wiley, New York, 1999), 3rd ed.

[12] R. Marqués, F. Mesa, J. Martel, and F. Medina, IEEE Trans. Antennas Propag. 51, 2572 (2003).

[13] G.V. Eleftheriades, O. Siddiqui, and A. K. Iyer, IEEE Microwave Wireless Compon. Lett. 13, 51 (2003).

[14] L. D. Landau, and E. M. Lifshitz, Electrodynamics of Continuous Media (Pergamon Press, Oxford, 1984), 2nd ed. 